http://jaet.journals.ekb.eg

\title{
Brain Tumor Automatic Detection from MRI Images Using Transfer Learning Model with Deep Convolutional Neural Network
}

\author{
Esraa Salah Bayoumi ${ }^{1}$, Mahmoud khaled Abd-Ellah ${ }^{* 2}$, Ashraf A. M. Khalaf ${ }^{3}$ \\ and Reda R. Gharieb ${ }^{4}$ \\ ${ }^{1}$ Electrical Engineering Department, Egyptian Academy for Engineering and Advanced Technology, Egypt \\ ${ }^{2}$ Electronics and Communications Department, Al-Madina Higher Institute for Engineering and Technology, Giza, \\ Egypt \\ 3 Electronics and Communications Department, Faculty of Engineering, Minia University, Egypt \\ 4 Electronics and Communications Department, Faculty of Engineering, Assiut University, Egypt
}

*corresponding author E-mail: mahmoudkhaled@ieee.org

\begin{abstract}
Brain tumor detection successfully in early-stage plays important role in improving patient treatment and survival. Evaluating magnetic resonance imaging (MRI) images manually is a very difficult task due to the numerous numbers of images produced in the clinic routinely. So, there is a need for using a computer-aided diagnosis (CAD) system for early detection and classification of brain tumors as normal and abnormal. The paper aims to design and evaluate the convolution neural network $(\mathrm{CNN})$ Transfer Learning state-of-the-art performance proposed for image classification over the recent years. Five different modifications have been applied to five different famous CNN to know the most effective modification. Five-layer modifications with parameter tuning are applied for each architecture providing a new CNN architecture for brain tumor detection. Most brain tumor datasets have a small number of images to train the deep learning structure. Therefore, two datasets are used in the evaluation to ensure the effectiveness of the proposed structures. Firstly, a standard dataset from the RIDER Neuro MRI database including 349 brain MRI images with 109 normal images and 240 abnormal images. Secondly, a collection of 120 brain MRI images including 60 abnormal images and 60 normal images. The results show that the proposed CNN Transfer Learning with MRI's can learn significant biomarkers of brain tumor, however, the best accuracy, specificity, and sensitivity gained is $100 \%$ for all of them.
\end{abstract}

Keywords: Brain Tumor, CNN Transfer Learning, Deep Learning, CNN, Tumor Classification.

\section{INTRODUCTION}

Brain tumor is proven to be a life-threatening and fatal disease [4]. In 2018, The American Cancer Society declared that there are 23,880 new instances with a brain tumor and 16,830 deaths in the United States alone [6]. Moreover, in recent decades, the number of died instances from brain tumors has increased in advanced nations by $300 \%$ as declared by the National Brain Tumor Foundation (NBTF) [7]. Accordingly, early diagnosis and treatment will lead to better results.

There are different brain imaging techniques that scan the structure and the function of the brain such as Electroencephalography (EEG), Position emission tomography (PET) and Magnetic resonance imaging (MRI). MRI provides better contrast to soft tissues than other techniques as it can present in detail and can distinguish between tissues in the brain. MRI images are considered safe for patients against harmful effects because it does not use any ionizing radiation during the examination [10].

Received:14 Septamber, 2020, Accepted: 21December, 2020
The radiologist comprehensively needs to manual analyze and evaluate a massive amount of MRI images in a short time which needs considerable effort and time. To assist the radiologist and doctors in proper diagnosis, computer-aided diagnosis (CAD) systems have been implemented [14]. CAD systems process digital images of typical appearances and focus completely on clear sections, such as the possibility of tumors, and then support the decision made by the specialist [14].

Machine learning (ML) needs data sets that have high quality and clear characteristics to be trained. To achieve the goal accurately and appropriately from ML, it must take time for the algorithms to learn and develop. ML can be mistaken a lot even though it is independent. Mistakes that occur from ML lead to another set of errors that are difficult to detect later for long periods. Moreover, when the source of the problem is discovered, this takes time, as well as time to correct it. Traditional ML algorithms for classification go through several stages such as preprocessing, feature extraction, feature reduction, and classification [11]. 


\section{Vol. 41, No.2. July 2022}

The artificial intelligence tools are represented in machine learning and deep learning. Nonetheless, The performance difference between deep learning and traditional machine learning makes the first more powerful. Deep learning has enormous neural networks that are trained many times for large data. This performance depends on supervised learning or on labeled data. The deep term refers to a large number of layers and the complex connections between layers. But there are some common drawbacks of deep learning such as it is complexity mechanized, requires a huge number of data to be enough for computations and training process of good algorithms and enhanced techniques. But these disadvantaged remains not confusing and continue improvements in training [22].

Recently, transfer learning becomes a supportive technique that depends on strong pre-trained CNN models to deal and solve various pattern problems [23]. Transfer learning is considered as a new model which transfer knowledge of pre-trained deep CNN model [24]. This technique tries to transfer the features which is learned from previous tasks and then apply those learned features to different objective tasks. The pros of transfer learning when compared to traditional deep learning is that: (i) a pre-trained model is used as an initial stage; (ii) a pre-trained model can be fine-tuned simpler and quicker than initializing a deep neural network from scratch[26]. In our study, different fine-tuning models are examined for each discussed pre-trained network to rate performances of brain tumor detection for each transferred architecture.

In this paper, we implement a special adjustment to the pre-trained network which can be combined structure modifications and tuning the parameters. Five pretrained networks are selected in this paper named AlexNet, Vgg16, GoogLeNet, Resnet50, and Inceptionv3. Five different structure modifications are applied to those networks providing 25 new structures. Those structures are compared to each other to get the best structure which compared to literature.

The remaining part of this manuscript is arranged as follows: Section 2 presents an overview of classical ML and Deep Learning algorithms in the literature. Section 3 provides the detailed methodology. Section 4 is about testing environment used in this paper. The results and discussion are explained in section 5. Finally, the conclusion and future work are given in section 6 .

\section{Literature}

For the last years, many types of research identify whether the MRI images are normal or abnormal. Javed et al.[1] used some texture features, invariant moments, and support vector machine (SVM) with multiclass. This technique classifies between normal and more than one class of abnormal images. The Database has 48 normal and 25 images abnormal for each abnormal class. Zacharaki et al. [2] presented a procedure to make the classification of various high and low glioma grades depending on SVMs and KNN. He attained an accuracy of $85 \%$ for the SVM classification system.

El-Dahshan et al. [3] proposed a system to classify brain tissues as normal and abnormal images. He used a Discrete Wavelet Transform (DWT) and Principal Component Analysis (PCA) for feature extraction and selection. Artificial neural network (ANN) and k-nearest neighbors (KNN) are used for classification, he obtained an accuracy of $97 \%$ and $98 \%$ respectively. Cheng et al. [5] performed a method to augment the classification of brain tumors by augmenting the tumor zone by image expansion and then by splitting into subzones. He used to extract features; Gray Level Co-occurrence Matrix (GLCM) and Bag of Words (BOW). Finally achieved the best accuracy of $91.28 \%$ by using ring form partition. M. K. Abd-Ellah et al. [30] detect brain tumor through MRI with machine learning model. The model used DWT and PCA for feature extraction and reduction. Then, SVM is applied for the classification.

Deep learning has made important strides in the field of machine learning and newly showed a noteworthy performance. In 2019, Deepak et al.[8] selected a pretrained deep neural network and applied the concept of transfer learning for brain tumor classification. They focused on the last three layers of the GoogLeNet and applied the modification. Then they trained this modified network and did the test by using the Softmax classifier. They also tried 2 other classifier models that are SVM and KNN. Here, they used the GoogLeNet as a feature extractor. They achieved a mean classification accuracy of $98 \%$.

In 2019, Swati et al.[31] concentrated on the power of deep learning about low-level and high-level features extraction. They used pre-trained VGG19 and adapt the idea of transfer learning with block wise fine-tuning to transfer the learning from original images to medical images especially the MRI images. Accuracy of 94.82 is obtained. Shahzadi et al. [12] proposed a cascade model consists of the CNN with long short term memory (LSTM) network which is another form of recurrent neural network (RNN) to classify the gliomas images into high and low grades. They relied on some deep networks as AlexNet, Vgg16 and ResNet with LSTM and stated an accuracy of $71 \%, 71 \%$ and $84 \%$ respectively.

In the research introduced by [17], the image classification of the brain CT was applied by using a deep neural network (DNN). They used a gray-level cooccurrence matrix (GLCM) to be a feature extractor for the classification done by DNN. They obtained an average accuracy of $83 \%$. Gao et al. [18] proposed an integrated 2D and 3D architectures of CNN to classify CT brain images. Each network contained seven layers. The average classification accuracy of $87.6 \%$ was achieved. Yan $\mathrm{Xu}$ et al. used deep convolutional activation features to classify and segment the brain tumor. The accuracy of $97.5 \%$ for this system classification was attained [21].

M. Toğaçar et al. proposed a new CNN named BrainMRNet to classify brain MR images. This module 


\section{Vol. 41, No.2. July 2022}

consisted of image preprocessing and augmentation, attention modules, convolutional layers with hypercolumn technique and dense layer before the classification stage. The accuracy of this model was obtained. Posteriorly, Kaur et al.[25] replaced last 3 layers for 8 pre-trained $\mathrm{CNN}$ such as: AlexNet, GoogLeNet, ResNet50, ResNet101, Vgg16, Vgg19, Inceptionv3 and InceptionResNetV2. They performed the

TABLE 1. An overview of techniques for brain tumor classification.

\begin{tabular}{|c|c|c|c|c|c|}
\hline Authors & Features Extraction & Classification & Dataset & $\begin{array}{l}\text { Ac } \\
(\%)\end{array}$ & Limitations \\
\hline [1] & $\begin{array}{l}\text { texture features and } \\
\text { invariant moments }\end{array}$ & SVM & 123 & - & - Not suitable for low contrast images. \\
\hline [2] & $\begin{array}{l}\text { Gabor texture } \\
\text { features. }\end{array}$ & $\begin{array}{l}\text { KNN and } \\
\text { SVM }\end{array}$ & 102 & $85 \%$ & $\begin{array}{l}\text { - Small dataset. } \\
\text { - Poor resulted accuracy. }\end{array}$ \\
\hline [3] & DWT and PCA & $\begin{array}{l}\mathrm{ANN} \text { and } \\
\mathrm{KNN}\end{array}$ & 70 & $98 \%$ & $\begin{array}{l}\text { - Few images are used. } \\
\text { - High computational complexity, cost, and } \\
\text { needs large storage. }\end{array}$ \\
\hline [5] & $\begin{array}{l}\text { Intensity histogram, } \\
\text { GLCM and BOW }\end{array}$ & $\begin{array}{l}\text { ring form } \\
\text { partition. }\end{array}$ & 3064. & $\begin{array}{l}91.28 \\
\%\end{array}$ & - High computational complexity. \\
\hline [9] & DWT and PCA & $\begin{array}{l}\text { Several } \\
\text { kernels of } \\
\text { SVM. }\end{array}$ & 349 & 66.96 & $\begin{array}{l}\text { - Small dataset. } \\
\text { - High computational cost and needs large } \\
\text { storage. }\end{array}$ \\
\hline [8] & Deep learning & $\begin{array}{l}\text { Softmax, } \\
\text { SVM and } \\
\text { KNN }\end{array}$ & 3064 & $98 \%$ & $\begin{array}{l}\text { - Dealing with a few number of layers for the } \\
\text { GoogLeNet. }\end{array}$ \\
\hline [11] & Deep learning & Softmax & 3064 & $94.8 \%$ & - High computational complexity. \\
\hline [12] & Deep learning & $\begin{array}{l}\text { Softmax and } \\
\text { LSTM }\end{array}$ & 120 & $84 \%$ & $\begin{array}{l}\text { - Have poor accuracy. } \\
\text { - Small dataset. }\end{array}$ \\
\hline [17] & GLCM & $\begin{array}{l}\text { Deep neural } \\
\text { network }\end{array}$ & 10 & $83 \%$ & $\begin{array}{l}\text { - Dataset is very small. } \\
\text { - Poor resulted accuracy. }\end{array}$ \\
\hline [18] & $\begin{array}{l}\text { Deep learning and } \\
\text { hand- crafted } \\
\text { features. }\end{array}$ & $\begin{array}{l}\text { Softmax and } \\
\text { SVM }\end{array}$ & 285 & $87.6 \%$ & -Poor resulted accuracy. \\
\hline [19] & Deep learning & SVM & 45 & $97.5 \%$ & - Not suitable for new training dataset. \\
\hline [20] & BrainMRNet model & $\begin{array}{l}\text { BrainMRNet } \\
\text { model }\end{array}$ & 253 & $96 \%$ & $\begin{array}{l}\text { - Time consuming. } \\
\text { - High computational complexity. }\end{array}$ \\
\hline [13] & Deep learning & Softmax & 253 & $95 \%$ & - Time consuming. \\
\hline [15] & Deep learning & Softmax & 253 & $97.2 \%$ & $\begin{array}{l}\text { - Time consuming. } \\
\text { - High computational complexity. }\end{array}$ \\
\hline [25] & Deep learning & Softmax & 160 & $95.9 \%$ & $\begin{array}{l}\text { - Time consuming. } \\
\text { - High computational complexity. }\end{array}$ \\
\hline [16] & Deep learning & Softmax & 3064 & $98.6 \%$ & $\begin{array}{l}\text { - Proposed method only classifies some brain } \\
\text { tumors (meningioma, glioma, and pituitary) } \\
\text { and does not detect it. }\end{array}$ \\
\hline [27] & Deep learning & ECOC-SVM. & 349 & $99.5 \%$ & $\begin{array}{l}\text { - High computational cost, complexity and } \\
\text { optimization. }\end{array}$ \\
\hline [28] & Deep learning & Softmax & 349 & 97.79 & - High computational complexity. \\
\hline [29] & Deep learning & Softmax & 1800 & $97.4 \%$ & - High computational complexity. \\
\hline
\end{tabular}

$96.05 \%$ [20]. Thereafter, Saxena et al.[13] applied pretrained vgg16, ResNet50 and Inceptionv3 networks to detect brain tumor cells as cancerous or noncancerous, based on transfer learning strategy. They performed preprocessing and augmentation techniques on the datasets. Best accuracy of $95 \%$ was obtained from ResNet50 network.

In 2020, Çinar et al.[15] used Resnet50 architecture to diagnose the tumor in the MRI images. They removed the last 5 layers of the Resnet50 and put other 8 layers. They also dealt with some pre-trained deep networks as AlexNet, GoogLeNet, DenseNet201 and Inceptionv3 for training and test the images. Best accuracy of $97.2 \%$ was training and testing by dividing the MRI datasets into $60 \%$ training and $40 \%$ testing. They achieved an accuracy of $94 \%$ and $95.92 \%$ from clinical, and benchmark Figshare repository datasets.

In another work, Rehman et al.[16] applied the classification of brain tumor by using 3 deep networks AlexNet, Vgg16 and GoogLeNet. Transfer learning is executed to extract most relevant features. They used preprocessing and augmentation to enhance and increase the database. The classification is done by two ways, which are Softmax and SVM classifier. Preferable accuracy of 98.69\% was attained from fine-tuned Vgg16. M. K. AbdEllah et al. presented a deep CNN model for brain tumor 
detection. The model extract features by $\mathrm{CNN}$ network and classify the extracted features by Error-correcting output coding-SVM (ECOC-SVM) [27]. M. K. AbdEllah et al. designed deep CNN architecture to detect brain tumor through MRI images [28]. They improved their model by designing a new CNN architecture for brain tumor detection and classification. The presented network used two parallel branches with two different filter size that extract both global and local features [29]. Different classification techniques are compared in Table 1 in terms of feature extraction, classification, Dataset, and performance. description of the pre-trained networks employed in our experiment is provided in Table 2. Those networks have been modified in five different ways. We start by modifying a number of layers each time for each network and see the effect on the accuracy. The detailed of each network modifications are provided in Fig 1 which includes designing new 5 transferred models for each deep CNN. This is done by replacing one layer in the first model and then replacing an additional layer in the second model, until reaching the last model, which contains five replaced layers. Then every model has trained and predicted by using softmax and classification layers. Finally the best model has accepted.
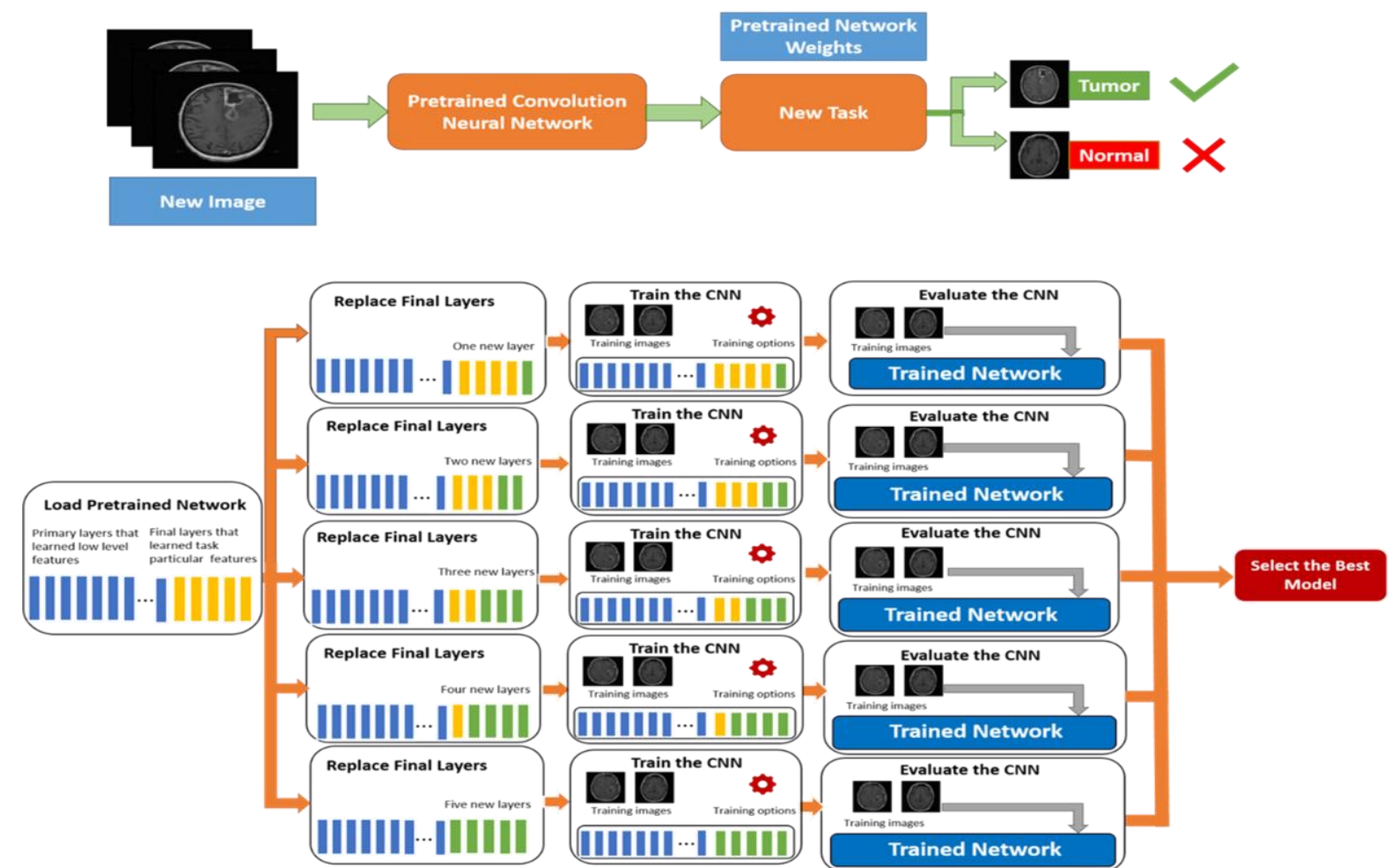

Fig 1. Flow chart of the proposed system. Upper part includes the main task of classification depending on transfer learning. Down part includes the applicable mechanism for the transferred layers in each model.

\section{METHODOLOGY}

The paper strategy suggests a more advanced systems, wherein special adjustments are implemented to the pre-trained network, to obtain the best results. Those adjustments may combine structure modifications and tuning the parameters. So, just distinct information gained from the prior task is maintained, whilst extra trainable parameters are included in the system. The new parameters need to be trained on a massive number of images to obtain advantageously. A brief

TABLE 2. The pretrained CNN and their parameters for transfer learning

\begin{tabular}{|c|c|c|c|}
\hline Layer name & DEPTH & PARAMETER & IMAGE INPUT SIZE \\
\hline AlexNet & 8 & $61 \mathrm{M}$ & 227-by-227 \\
\hline Vgg16 & 16 & $138 \mathrm{M}$ & 224-by-224 \\
\hline GoogLeNet & 22 & $7 M$ & 224-by-224 \\
\hline Resnet50 & 50 & $25.6 \mathrm{M}$ & 224-by-224 \\
\hline Inceptionv3 & 48 & $23.9 \mathrm{M}$ & 299-by-299 \\
\hline
\end{tabular}

\subsection{Pre-processing}

Before starting the proposed structure, a pre-processing step is obtained. To get a better performance in lower time and more unpretentious calculations of the network, the original image dimensions must be decreased by downing-size the images [32]. First database (349 samples) has original gray images with size of $256 \times 256 \times 1$ pixels, where second database $(120$ samples) has original gray images with size of $512 \times 512 \times 1$ pixels. The original images of both database are downed size into $227 \times 227 \times 1$ for (AlexNet), $224 \times 224 \times 1$ for $(\mathrm{Vgg} 16$, GoogLeNet and Resnet50) and $299 \times 299 \times 1$ for Inceptionv3. Then the resulted gray images are converted to RGB images to be compatible with input layer of each deep network. 
Vol. 41, No.2. July 2022

TABLE 3. Architecture Of AlexNet

\begin{tabular}{|c|c|c|c|}
\hline Layer & $\begin{array}{l}\text { Layer } \\
\text { function }\end{array}$ & $\begin{array}{l}\text { Output } \\
\text { shape }\end{array}$ & $\begin{array}{l}\text { Learnable } \\
\text { parameters }\end{array}$ \\
\hline 1 & Image Input & {$[227,227,3]$} & 0 \\
\hline 2 & Convolution & {$[55,55,96]$} & 34,944 \\
\hline 3 & ReLU & {$[55,55,96]$} & 0 \\
\hline 4 & $\begin{array}{l}\text { Cross Channel } \\
\text { Normalization }\end{array}$ & {$[55,55,96]$} & 0 \\
\hline 5 & Max Pooling & {$[27,27,96]$} & 0 \\
\hline 6 & $\begin{array}{l}\text { Grouped } \\
\text { Convolution }\end{array}$ & {$[27,27,256]$} & 307,456 \\
\hline 7 & ReLU & {$[27,27,256]$} & 0 \\
\hline 8 & $\begin{array}{l}\text { Cross Channel } \\
\text { Normalization }\end{array}$ & {$[27,27,256]$} & 0 \\
\hline 9 & Max Pooling & {$[13,13,256]$} & 0 \\
\hline 10 & Convolution & {$[13,13,384]$} & 885,120 \\
\hline 11 & $\operatorname{ReLU}$ & {$[13,13,384]$} & 0 \\
\hline 12 & Convolution & {$[13,13,384]$} & 663,936 \\
\hline 13 & ReLU & {$[13,13,384]$} & 0 \\
\hline 14 & Convolution & {$[13,13,256]$} & 442,624 \\
\hline 15 & ReLU & {$[13,13,256]$} & 0 \\
\hline 16 & Max Pooling & {$[6,6,256]$} & 0 \\
\hline 17 & $\begin{array}{l}4096 \text { fully } \\
\text { Connected }\end{array}$ & {$[1,1,4096]$} & $37,752,832$ \\
\hline 18 & ReLU & {$[1,1,4096]$} & 0 \\
\hline 19 & Dropout & {$[1,1,4096]$} & 0 \\
\hline 20 & $\begin{array}{l}4096 \text { fully } \\
\text { Connected }\end{array}$ & {$[1,1,4096]$} & $16,781,312$ \\
\hline 21 & ReLU & {$[1,1,4096]$} & 0 \\
\hline 22 & Dropout & {$[1,1,4096]$} & 0 \\
\hline 23 & $\begin{array}{l}1000 \text { fully } \\
\text { Connected }\end{array}$ & {$[1,1,1000]$} & $4,097,000$ \\
\hline 24 & Softmax & {$[1,1,1000]$} & 0 \\
\hline 25 & $\begin{array}{l}\text { Classification } \\
\text { Output }\end{array}$ & - & 0 \\
\hline
\end{tabular}

\subsection{CNNs Transfer learning}

Transfer learning transfer the collected knowledge from the dataset by $\mathrm{CNN}$ to resolve another related task, including a new dataset, that contains an insufficient number of samples to train the network from the scratch.

\subsubsection{Transferred AlexNet Models}

Five models depend on the employment of a famous deep learning-based CNN named AlexNet. The utilized parameters are tabulated in Table 3 that contains 25 layers. The first layer in all models is the input layer which has a fixed size of 227-by-227-by-3 pixels that is an RGB image. Each model has a number of changed layers. Accordingly, number of parameters of each model has also changed from the original number of parameters $(61 \mathrm{M})$. The brief explanation of all models' layer modifications is tabulated in Table 4. The unchanged layers are a portion of the pre-trained AlexNet network which trained with another dataset named ImageNet.

\subsubsection{Transferred Vgg16 Models}

Five models depend on the employment of a famous deep learning-based CNN named Vgg16. The first layer in all models is the input layer which has a fixed size of 224-by-224-by-3 pixels that is an RGB image. Each model has a number of changed layers.
Accordingly, the total parameters value of each model has also changed from the original value of total parameters $(138 \mathrm{M})$. The brief explanation of all models' layer modifications is tabulated in Table 5.

TABLE 5. The transferred VGG16 models and their output.

\begin{tabular}{llllll}
\hline \hline Model & LAYER & OLD & NEW & SHAPE & PARAMETER \\
\hline Model 1 & 39 & 1000 FC & 2 FC & {$[1,1,2]$} & $134.3 \mathrm{M}$ \\
\hline \multirow{2}{*}{ Model 2 } & 38 & Dropout & ReLU & {$[1,1,30]$} & \multirow{2}{*}{$134.3 \mathrm{M}$} \\
& 39 & 1000 FC & 2 FC & {$[1,1,2]$} & \\
\hline \multirow{3}{*}{ Model 3 } & 37 & Relu & FC & {$[1,1,30]$} & \\
& 38 & Dropout & $\operatorname{ReLU}$ & {$[1,1,30]$} & $134.4 \mathrm{M}$ \\
& 39 & 1000 FC & 2 FC & {$[1,1,2]$} & \\
\hline & 36 & 4096 FC & CONV & {$[1,1,8]$} & \\
Model 4 & 37 & Relu & FC & {$[1,1,30]$} & \multirow{2}{*}{$118.5 \mathrm{M}$} \\
& 38 & Dropout & $\operatorname{ReLU}$ & {$[1,1,30]$} & \\
& 39 & 1000 FC & 2 FC & {$[1,1,2]$} & \\
\hline & 35 & Dropout & BN & {$[1,1,4096]$} & \\
& 36 & 4096 FC & CONV & {$[1,1,8]$} & \\
Model 5 & 37 & Relu & FC & {$[1,1,30]$} & $118.5 \mathrm{M}$ \\
& 38 & Dropout & ReLU & {$[1,1,30]$} & \\
& 39 & 1000 FC & 2 FC & {$[1,1,2]$} & \\
\hline \hline
\end{tabular}

\subsubsection{Transferred GoogLeNet Models}

Five models depend on the employment of a famous deep learning-based CNN named GoogLeNet. The first layer in all models is the input layer which has a fixed size of 224-by-224-by-3 pixels that is an RGB image. Each model has a number of changed layers. Accordingly, the total parameters value of each model has also changed from the original value of total parameters $(7 \mathrm{M})$. The brief explanation of all models' layer modifications is tabulated in Table 6.

TABLE 6. The transferred GOOGLENET models and their output.

\begin{tabular}{|c|c|c|c|c|c|c|c|}
\hline Model & LA) & YER & LD & & $\mathrm{SH}$ & & ARAMETE \\
\hline Model & LAYER & $\overline{O L D}$ & & NEW & SHAPE & PARAMETER & \\
\hline Model 1 & 23 & 100 & $0 \mathrm{FC}$ & $2 \mathrm{FC}$ & {$[1,1,2]$} & $56.9 \mathrm{M}$ & \\
\hline Model $?$ & 22 & Dro & pout & ReLU & {$[1,1,30]$} & $560 \mathrm{M}$ & \\
\hline IVioder 2 & 23 & 100 & $0 \mathrm{FC}$ & $2 \mathrm{FC}$ & {$[1,1,2]$} & $50.9 \mathrm{IVI}$ & \\
\hline & 21 & Rel & & FC & {$[1,1,30]$} & & \\
\hline Model 3 & 22 & Dro & pout & ReLU & {$[1,1,30]$} & $57 \mathrm{M}$ & \\
\hline & 23 & 100 & $0 \mathrm{FC}$ & $2 \mathrm{FC}$ & {$[1,1,2]$} & & \\
\hline & 20 & 409 & $6 \mathrm{FC}$ & CONV & {$[1,1,8]$} & & \\
\hline A d d & 21 & Rel & & FC & {$[1,1,30]$} & 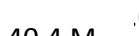 & $.6 \mathrm{M}$ \\
\hline Miodel 4 & 22 & Dro & pout & ReLU & {$[1,1,30]$} & $40.4 \mathrm{IV}$ & \\
\hline & 23 & 100 & $0 \mathrm{FC}$ & $2 \mathrm{FC}$ & {$[1,1,2]$} & & \\
\hline & 19 & Dro & pout & $\mathrm{BN}$ & {$[1,1,4096]$} & & \\
\hline & 20 & 409 & $6 \mathrm{FC}$ & CONV & {$[1,1,8]$} & & \\
\hline Model 5 & 21 & Rel & & $\mathrm{FC}$ & {$[1,1,30]$} & $40.4 \mathrm{M}$ & \\
\hline & 22 & Dro & pout & ReLU & {$[1,1,30]$} & & $.6 \mathrm{M}$ \\
\hline & 23 & 100 & $0 \mathrm{FC}$ & $2 \mathrm{FC}$ & {$[1,1,2]$} & & \\
\hline & $\overline{14}$ & & Dropout & & $\overline{11,}$ & $1,30 \mathrm{~J}$ & \\
\hline & 14 & & $1000 \mathrm{FC}$ & & {$[1$,} & $1,2]$ & \\
\hline & & & -- & & - & i-, - - & \\
\hline & Mode & & 21 & & Connected & {$[1,1,30]$} & \\
\hline & & & 22 & & & {$[1,1,30]$} & \\
\hline & & & 23 & & Connected & {$[1,1,2]$} & \\
\hline & & & 19 & & malization & {$[1,1,409$} & \\
\hline & Mode & & 20 & & volution & {$[1,1,8]$} & \\
\hline & IVIode & & 21 & & Connected & {$[1,1,30]$} & \\
\hline & & & 22 & & & {$[1,1,30]$} & \\
\hline & & & 23 & & Connected & {$[1,1,2]$} & \\
\hline
\end{tabular}




\subsubsection{Transferred Resnet50 Models}
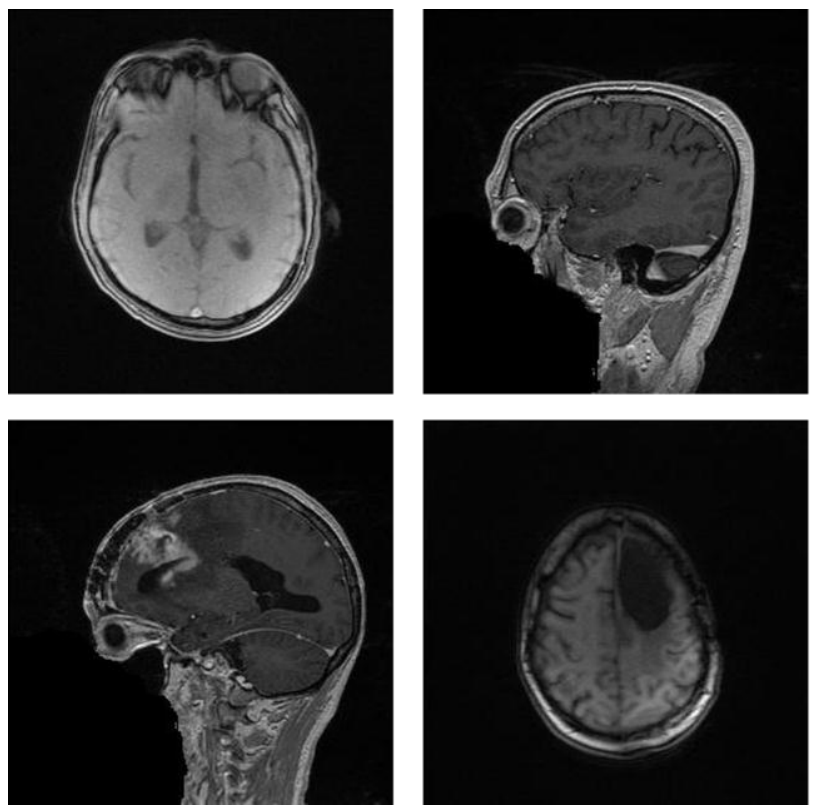

Accordingly, the total parameters value of each model
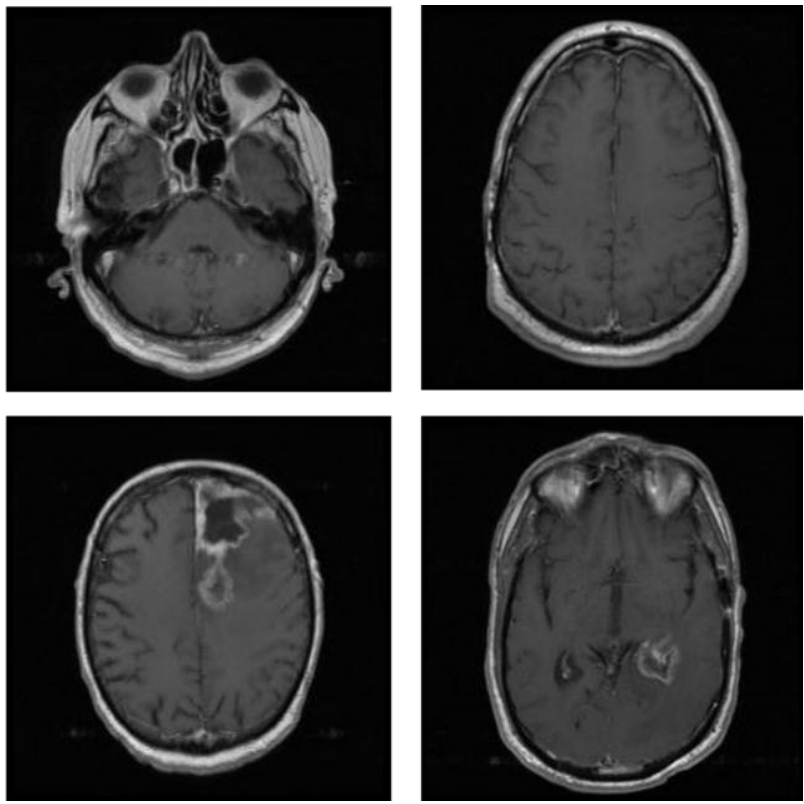

Fig2. Sampled images from the Two datasets. The normal images are in the first row, while abnormal images are in the second raw. The first two-columns from the left belongs to the first dataset (RIDER dataset), while the other two-column from the

TABLE 7. The transferred RESNET50 models and their output.

\begin{tabular}{|c|c|c|c|c|c|}
\hline Model & LAYER & OLD & NEW & SHAPE & PARAMETER \\
\hline Model 1 & 175 & $1000 \mathrm{FC}$ & $2 \mathrm{FC}$ & {$[1,1,2]$} & $23.5 \mathrm{M}$ \\
\hline \multirow[t]{2}{*}{ Model 2} & 174 & $\begin{array}{l}\text { Average } \\
\text { Pooling }\end{array}$ & ReLU & {$[1,1,30]$} & \multirow[t]{2}{*}{$23.7 \mathrm{M}$} \\
\hline & 175 & $1000 \mathrm{FC}$ & $2 \mathrm{FC}$ & {$[1,1,2]$} & \\
\hline \multirow{3}{*}{ Model 3} & 173 & ReLU & FC & {$[1,1,30]$} & \multirow{3}{*}{$25.5 \mathrm{M}$} \\
\hline & 174 & $\begin{array}{l}\text { Average } \\
\text { Pooling }\end{array}$ & ReLU & {$[1,1,30]$} & \\
\hline & 175 & $1000 \mathrm{FC}$ & $2 \mathrm{FC}$ & {$[1,1,2]$} & \\
\hline \multirow{4}{*}{ Model 4} & 172 & Addition & Concatenate & {$[1,1,8]$} & \multirow{4}{*}{$27.5 \mathrm{M}$} \\
\hline & 173 & ReLU & FC & {$[1,1,30]$} & \\
\hline & 174 & $\begin{array}{l}\text { Average } \\
\text { Pooling }\end{array}$ & ReLU & {$[1,1,30]$} & \\
\hline & 175 & $1000 \mathrm{FC}$ & $2 \mathrm{FC}$ & {$[1,1,2]$} & \\
\hline \multirow{5}{*}{ Model 5} & 171 & $\mathrm{BN}$ & CONV & {$[1,1,4096]$} & \multirow{5}{*}{$26.1 \mathrm{M}$} \\
\hline & 172 & Addition & Concatenate & {$[1,1,8]$} & \\
\hline & 173 & ReLU & $\mathrm{FC}$ & {$[1,1,30]$} & \\
\hline & 174 & $\begin{array}{l}\text { Average } \\
\text { Pooling }\end{array}$ & ReLU & {$[1,1,30]$} & \\
\hline & 175 & $1000 \mathrm{FC}$ & $2 \mathrm{FC}$ & {$[1,1,2]$} & \\
\hline
\end{tabular}

Five models depend on the employment of a famous deep learning-based CNN named Resnet50. The first layer in all models is the input layer which has a fixed size of 224-by-224-by-3 pixels that is an RGB image. Each model has a number of changed layers. Accordingly, the total parameters value of each model has also changed from the original value of total parameters $(25.6 \mathrm{M})$. The brief explanation of all models' layer modifications is tabulated in Table 7.

\subsubsection{Transferred Inceptionv3 Models}

Five models depend on the employment of a famous deep learning-based CNN named Inceptionv3. The first layer in all models is the input layer which has a fixed size of 299-by-299-by-3 pixels that is an RGB image. Each model has a number of changed layers.
TABLE 8. The transferred INCEPTIONV3 models and their output.

\begin{tabular}{|c|c|c|c|c|c|}
\hline Model & LAYER & OLD & NEW & SHAPE & PARAMETER \\
\hline Model 1 & 314 & $1000 \mathrm{FC}$ & $2 \mathrm{FC}$ & {$[1,1,2]$} & $21.8 \mathrm{M}$ \\
\hline \multirow{2}{*}{ Model 2} & 313 & $\begin{array}{l}\text { Average } \\
\text { Pooling }\end{array}$ & ReLU & {$[1,1,30]$} & \multirow{2}{*}{$22.1 \mathrm{M}$} \\
\hline & 314 & $1000 \mathrm{FC}$ & $2 \mathrm{FC}$ & {$[1,1,2]$} & \\
\hline \multirow{3}{*}{ Model 3} & 307 & $\mathrm{BN}$ & CONV & {$[1,1,30]$} & \multirow{3}{*}{$23 \mathrm{M}$} \\
\hline & 313 & $\begin{array}{l}\text { Average } \\
\text { Pooling }\end{array}$ & ReLU & {$[1,1,30]$} & \\
\hline & 314 & $1000 \mathrm{FC}$ & $2 \mathrm{FC}$ & {$[1,1,2]$} & \\
\hline \multirow{4}{*}{ Model 4} & 300 & $\mathrm{BN}$ & CONV & {$[1,1,8]$} & \multirow{4}{*}{$26.7 \mathrm{M}$} \\
\hline & 307 & $\mathrm{BN}$ & CONV & {$[1,1,30]$} & \\
\hline & 313 & $\begin{array}{l}\text { Average } \\
\text { Pooling }\end{array}$ & ReLU & {$[1,1,30]$} & \\
\hline & 314 & $1000 \mathrm{FC}$ & $2 \mathrm{FC}$ & {$[1,1,2]$} & \\
\hline \multirow{5}{*}{ Model 5} & 299 & $\mathrm{BN}$ & CONV & {$[1,1,4096]$} & \multirow{5}{*}{$28 \mathrm{M}$} \\
\hline & 300 & $\mathrm{BN}$ & CONV & {$[1,1,8]$} & \\
\hline & 307 & $\mathrm{BN}$ & CONV & {$[1,1,30]$} & \\
\hline & 313 & $\begin{array}{l}\text { Average } \\
\text { Pooling }\end{array}$ & ReLU & {$[1,1,30]$} & \\
\hline & 314 & $1000 \mathrm{FC}$ & $2 \mathrm{FC}$ & {$[1,1,2]$} & \\
\hline
\end{tabular}

has also changed from the original value of total parameters $(23.9 \mathrm{M})$. The brief explanation of all models' layer modifications is tabulated in Table 8 .

\section{TESTING ENVIRONMENT}

\subsection{Machine tool}

The specifications of the device in which the work was carried out included the following: processor of intel(R) Core (TM) i7-4500U CPU @ $1.80 \mathrm{GHz} 2.40 \mathrm{GHz}$. Intel(R) HD Graphics Family. RAM of $8 \mathrm{~GB}$ and operating system of 64 bit. The software of the proposed system computations was implemented on Matlab R2019a.

\subsection{Dataset}

The first database was acquired from a standard dataset 
Vol. 41, No.2. July 2022

named RIDER Neuro MRI database. It was acquired from 19 patients with T1-weighted and T2-weighted MRIs which includes 349 brain MRI images with 109 normal images and 240 abnormal images. The second

Table 9. Training and testing data

\begin{tabular}{llllll}
\hline \hline \multirow{2}{*}{ Dataset } & \multicolumn{2}{l}{ Training data } & \multicolumn{2}{l}{ Testing data } & Total \\
\cline { 2 - 5 } & Tumor & Normal & Tumor & Normal & \\
\hline Dataset 1 & 77 & 45 & 163 & 64 & 349 \\
Dataset 2 & 42 & 42 & 18 & 18 & 120 \\
\hline \hline
\end{tabular}

database was acquired from Brain-Tumor-Progression. All image sets are in DICOM format and contain T1weighted (pre-contrast and post-contrast agent), acquired from 20 patients with 6 slices. The second dataset was divided into two parts training sets $(70 \%)$ and test sets $(30 \%)$. Sampled MRI images from the datasets are presented in Fig 2. Details are shown in Table 9. The two datasets are published in TCIA (The Cancer Imaging Archive) with the last updated version in 2020 [33].

\section{EXPERIMENTAL RESULTS AND DISSCUSSION}

\subsection{Evaluation metric}

To summarize the prediction results, Sensitivity, Specificity, and Accuracy have been calculated from confusion matrix's parameters in equations:

$$
\begin{gathered}
\text { Specificity }(\mathrm{Sp})=\frac{T N}{T N+F P} \\
\text { Sensitivity (SV) }=\frac{T P}{T P+F N} \\
\text { Accuracy }(\text { Acc })=\frac{T P+T N}{P+N}
\end{gathered}
$$

Where, true negative (TN) is number of normal predicted samples and they are also actually normal.

Table 10. Tested hyper-parameters

\begin{tabular}{lc}
\hline \multicolumn{1}{c}{ Parameter } & Number \\
\hline Convolutional layer & $1,2,3$ \\
ReLU layer & 1,2 \\
Batch normalization layer & 1,2 \\
concatenation layer & 1 \\
Fully connected layer & $1,2,3$ \\
Mini-batch size & $8,16,32$ \\
Maximum epochs & $5,7,40,60,80,100,140,160$ \\
Initial learning rate & $0.01,0.001,0.0001$ \\
Learning frequency & $1,2,3,8$ \\
\hline \hline
\end{tabular}

True positive (TP) is number of tumors predicted samples and they are also actually tumor. False negative (FN) is the number of normal predicted samples while they are actually tumor. False positive is the number of tumor samples while they are actually

\begin{tabular}{|c|c|c|c|c|c|c|c|c|c|}
\hline \multirow{2}{*}{$\begin{array}{c}\text { Network } \\
\text { Name }\end{array}$} & \multicolumn{8}{|c|}{ DATASET 1} & \multirow{2}{*}{$\begin{array}{l}\text { DATASET } 2 \\
\text { Accuracy }\end{array}$} \\
\hline & Model & TP & TN & FP & FN & Accuracy & Sensitivity & Specificity & \\
\hline \multirow{5}{*}{ AlexNet } & Model 1 & 63 & 163 & 1 & 0 & $99.56 \%$ & $100.00 \%$ & $99.39 \%$ & $97.22 \%$ \\
\hline & Model 2 & 60 & 163 & 4 & 0 & $98.24 \%$ & $100.00 \%$ & $97.60 \%$ & $91.67 \%$ \\
\hline & Model 3 & 61 & 161 & 3 & 2 & $97.80 \%$ & $96.83 \%$ & $98.17 \%$ & $100 \%$ \\
\hline & Model 4 & 63 & 162 & 1 & 1 & $99.12 \%$ & $98.44 \%$ & $99.39 \%$ & $100 \%$ \\
\hline & Model 5 & 47 & 150 & 17 & 13 & $86.78 \%$ & $78.33 \%$ & $89.82 \%$ & $91.67 \%$ \\
\hline \multirow{5}{*}{ Vgg16 } & Model 1 & 64 & 59 & 0 & 104 & $54.19 \%$ & $38.10 \%$ & $100.00 \%$ & $91.67 \%$ \\
\hline & Model 2 & 60 & 113 & 4 & 50 & $76.21 \%$ & $54.55 \%$ & $96.58 \%$ & $88.89 \%$ \\
\hline & Model 3 & 62 & 97 & 2 & 66 & $70.04 \%$ & $48.44 \%$ & $97.98 \%$ & $86.11 \%$ \\
\hline & Model 4 & 45 & 162 & 19 & 1 & $91.19 \%$ & $97.83 \%$ & $89.50 \%$ & $97.22 \%$ \\
\hline & Model 5 & 54 & 134 & 10 & 29 & $82.82 \%$ & $65.06 \%$ & $93.06 \%$ & $69.44 \%$ \\
\hline \multirow{5}{*}{ GoogLeNet } & Model 1 & 61 & 137 & 3 & 26 & $87.22 \%$ & $70.11 \%$ & $97.86 \%$ & $97.22 \%$ \\
\hline & Model 2 & 59 & 156 & 5 & 7 & $94.71 \%$ & $89.39 \%$ & $96.89 \%$ & $94.44 \%$ \\
\hline & Model 3 & 0 & 163 & 64 & 0 & $71.81 \%$ & NAN & $71.81 \%$ & $88.89 \%$ \\
\hline & Model 4 & 0 & 163 & 64 & 0 & $71.81 \%$ & NAN & $71.81 \%$ & $94.44 \%$ \\
\hline & Model 5 & 12 & 163 & 52 & 0 & $77.09 \%$ & $100.00 \%$ & $75.81 \%$ & $94.44 \%$ \\
\hline \multirow{5}{*}{ Resnet50 } & Model 1 & 44 & 161 & 20 & 2 & $90.31 \%$ & $95.65 \%$ & $88.95 \%$ & $91.67 \%$ \\
\hline & Model 2 & 56 & 148 & 8 & 15 & $89.87 \%$ & $78.87 \%$ & $94.87 \%$ & $91.67 \%$ \\
\hline & Model 3 & 0 & 163 & 64 & 0 & $71.81 \%$ & NAN & $71.81 \%$ & $94.44 \%$ \\
\hline & Model 4 & 0 & 163 & 64 & 0 & $71.81 \%$ & NAN & $71.81 \%$ & $91.67 \%$ \\
\hline & Model 5 & 0 & 163 & 64 & 0 & $71.81 \%$ & NAN & $71.81 \%$ & $97.22 \%$ \\
\hline \multirow{5}{*}{ Inceptionv3 } & Model 1 & 42 & 158 & 22 & 5 & $88.11 \%$ & $89.36 \%$ & $87.78 \%$ & $80.56 \%$ \\
\hline & Model 2 & 64 & 125 & 0 & 38 & $83.26 \%$ & $62.75 \%$ & $100.00 \%$ & $83.33 \%$ \\
\hline & Model 3 & 62 & 108 & 2 & 55 & $74.89 \%$ & $52.99 \%$ & $98.18 \%$ & $86.11 \%$ \\
\hline & Model 4 & 54 & 155 & 10 & 8 & $92.07 \%$ & $87.10 \%$ & $93.94 \%$ & $86.11 \%$ \\
\hline & Model 5 & 61 & 141 & 3 & 22 & $88.99 \%$ & $73.49 \%$ & $97.92 \%$ & $94.44 \%$ \\
\hline
\end{tabular}

TABLE 11. Comparison of all proposed transfer learning models with the two datasets. The highest performance of the models has been fulfilled by using the bolding architectures. 
Vol. 41, No.2. July 2022

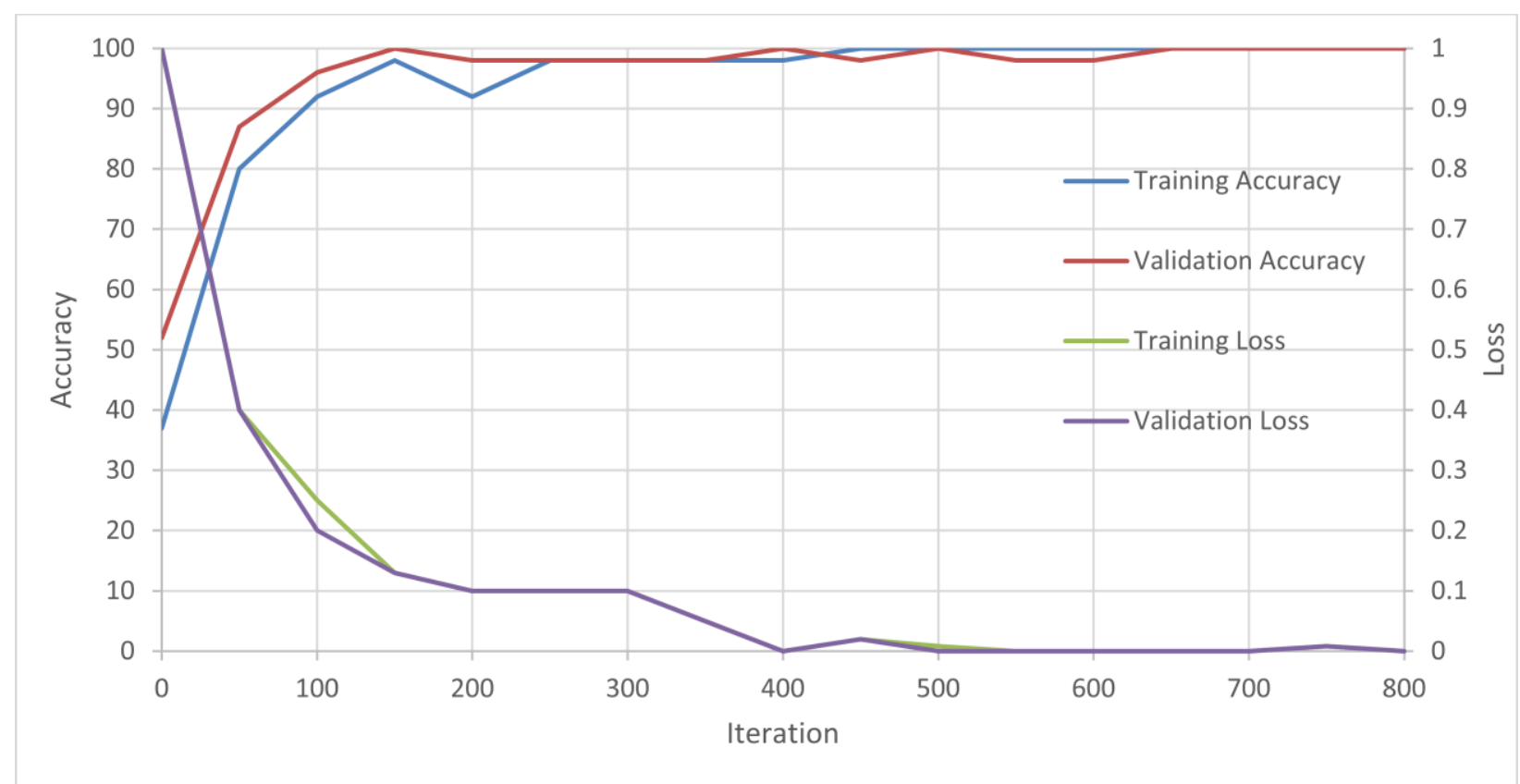

Fig 3. Training and testing progress for the best proposed transfer learning Model.

normal. $\mathrm{N}$ is the number of normal cases while $\mathrm{P}$ is the number of tumor cases.

\subsection{Hyper-parameter optimization}

The tuning is an essential step in selecting a group of hyper-parameters for a learning algorithm. The optimized hyper-parameters have a great impact on network performance. In this section, the various architectures parameters in each proposed model are presented. Table 10 shows a different number of layers and hyper-parameters that were tested before reaching the effective performance for each model of all CNNs.

\subsection{Results}

Table 11 shows a comparison between all proposed

Table 12. Comparison between proposed system and other related works.

\begin{tabular}{lccc}
\hline \hline \multicolumn{1}{c}{ Method } & Image & $\begin{array}{c}\text { ACC } \\
\text { \% }\end{array}$ & $\begin{array}{c}\text { Classification } \\
\text { method }\end{array}$ \\
\hline Zacharaki et al.[2] & 102 & 85 & SVM and KNN \\
El-Dahshan et al.[3] & 70 & 98 & ANN and KNN \\
Cheng et al.[5] & 3064 & 91 & SVM and KNN \\
Deepak et al.[8] & 3064 & 98 & SVM and KNN \\
Swati et al.[11] & 3064 & 94.82 & CNN \\
Shahzadi et al.[12] & 120 & 84 & CNN and LSTM \\
Saxena et al.[13] & 253 & 95 & CNN \\
Çinar et al.[15] & 253 & 97.2 & CNN \\
Rehman et al.[16] & 3064 & 98.69 & CNN \\
Da et al. [17] & 10 & 83.0 & DNN \\
Gao et al. [18] & 285 & 87.6 & 2D, 3D CNNs \\
Yan Xu et al. [21] & 45 & 97.50 & CNNs \\
Toğaçar et al. [20] & 253 & 96.05 & BrainMRNet \\
Kaur et al.[25] & 74 & 94 & Pre-trained CNN \\
& 160 & 95.92 & \\
Proposed system & 349 & $\mathbf{9 9 . 5 6}$ & Pre-trained CNN \\
\hline \hline
\end{tabular}

Table 13. Comparison between proposed system to previous work used with the same database as a unified benchmark.

\begin{tabular}{llllll}
\hline \hline Method & Image & Sp \% & Sv \% & Acc \% & $\begin{array}{l}\text { Classification } \\
\text { method }\end{array}$ \\
\hline$[9]$ & 349 & $25.0 \%$ & $83.43 \%$ & $66.96 \%$ & SVM \\
{$[27]$} & 349 & $100.0 \%$ & $99.38 \%$ & $99.5 \%$ & ECOC-SVM \\
{$[28]$} & 349 & $97.54 \%$ & $98.43 \%$ & $97.79 \%$ & Softmax \\
$\begin{array}{l}\text { Proposed } \\
\text { Method }\end{array}$ & $\mathbf{3 4 9}$ & $\mathbf{9 9 . 3 9 \%}$ & $\mathbf{1 0 0 \%}$ & $\mathbf{9 9 . 5 6 \%}$ & Softmax \\
\hline \hline
\end{tabular}

transfer learning models for each deep CNN network. Confusion matrix parameters, Accuracy, Sensitivity, and Specificity are obtained for the first datasets while the accuracy is attained for the second datasets. As shown, there are different results that appear the effectiveness of the models, while the best models have resulted from AlexNet. These models are model 3 and model 4, which have the same best Accuracy, Sensitivity, and Specificity of $100 \%$.

Table 12 shows a comparison between our proposed system and the other state-of-the-arts who's applied different machine learning and deep learning architectures for brain tumor classification presented in the literature. In addition, table 13 displays a detailed comparison between our work and other previous works that have the same first database. We must always be mindful that a false negative in this data is deciding that patients with a tumor are told that they may not want to pursue further treatment, allowing the tumor to progress quietly. Further, A false positive is deciding that a tumor-free patient is told that warrants further invasive tests and/or treatment. The best model can be clarified as the model that gets false-negative 
equal to zero and Sensitivity equal to $100 \%$, which shows the proposed model's superiority over other literature models.

The result of the best-proposed model is compared with other related works that pretend the power of the proposed deep CNN structure, where our method has overcome some faults that were present in literature as computational complexity and hand-crafted features. Our proposed system is considered accurate due to the appropriate transferred layers that learned the optimized features. Moreover, the best resulted models (model 3 and 4 of AlexNet) have reduced weights (57 M, $40.4 \mathrm{M})$ than pre-trained AlexNet model (61M). This means that the network capacity is simpler in computations and runtime.

Figure 3 shows the outcomes of accuracy and loss rates for training and testing progress of our best proposed fine-tuned model 3 of the AlexNet. This figure gives us some important notes: The training and testing loss both reach the minimum value at the end, which is very close to zero. Likewise, the training and testing accuracy both reach the maximum value at the end of iterations. Although the data set is small, we find the best accuracy and loss rate and this is the benefit of using Transfer Learning. The mini-batch size is set to 16 and the number of iterations at 800 .

\subsection{Discussion}

In this paper, a procedure for brain tumor classification is proposed by modifying convolution neural network models based on the transfer learning algorithm with magnetic resonance images. The proposed transfer learning system can train a deep $\mathrm{CNN}$ for more than one dataset. Large collection of parameters is used to adjust the 25 different model systems to reach an effective result for each of the proposed models for the 5 CNNs. Training a fine-tuned CNN readily with a small dataset is challenging as it maybe takes a time to achieve acceptable results for a database without being over conform or under conform. Another important portion is the difference in performance between traditional machine learning and deep transfer learning.

\section{CONCLUSION AND FUTURE WORK}

In this paper, a detailed study about the classification is discussed for five modifications to five different CNNs. The deep learning model depending on a finetuning convolutional neural network to classify brain tissues as normal and abnormal sets. Each proposed model consists of different layers beginning with the input layer until the last output layer. For all proposed models, the softmax layer is used for classification to provide the predicted class. The experimental results of our structures propose an accuracy of $100.00 \%$ for the best two AlexNet models. In future work, we will deal with multi-class for brain tumors classification.

\section{References}

[1] U. Javed, M. Riaz, A. Ghafoor, and T. Cheema, "MRI brain classification using texture features, fuzzy weighting and support vector machine," Progress In Electromagnetics Research B, vol. 53, pp. 73-88, 01/01, 2013.

[2] E. I. Zacharaki, S. Wang, S. Chawla, D. Soo Yoo, R. Wolf, E. R. Melhem, and C. Davatzikos, "Classification of brain tumor type and grade using MRI texture and shape in a machine learning scheme," Magnetic Resonance in Medicine, vol. 62, no. 6, pp. 1609-1618, 2009.

[3] E.-S. A. El-Dahshan, T. Hosny, and A.-B. M. Salem, "Hybrid intelligent techniques for MRI brain images classification," Digital Signal Processing, vol. 20, no. 2, pp. 433441, Mar., 2010.

[4] S. Kumar, C. Dabas, and S. Godara, "Classification of Brain MRI Tumor Images: A Hybrid Approach," Procedia Computer Science, vol. 122, pp. 510-517, 01/01, 2017.

[5] J. Cheng, W. Huang, S. Cao, R. Yang, W. Yang, Z. Yun, Z. Wang, and Q. Feng, "Correction: Enhanced Performance of Brain Tumor Classification via Tumor Region Augmentation and Partition," PLOS ONE, vol. 10, no. 12, pp. e0144479, 2015.

[6] M. K. Abd-Ellah, A. A. M. Khalaf, A. I. Awad, and H. F. A. Hamed, "TPUAR-Net: Two Parallel U-Net with Asymmetric Residual-Based Deep Convolutional Neural Network for Brain Tumor Segmentation." pp. 106-116.

[7] M. K. Abd-Ellah, A. I. Awad, A. A. M. Khalaf, and H. F. A. Hamed, "Design and implementation of a computer-aided diagnosis system for brain tumor classification." pp. 73-76.

[8] S. Deepak, and P. M. Ameer, "Brain tumor classification using deep $\mathrm{CNN}$ features via transfer learning," Computers in Biology and Medicine, vol. 111, pp. 103345, 2019/08/01/, 
2019.

[9] M. K. Abd-Ellah, A. I. Awad, A. A. Khalaf, and H. F. Hamed, "Classification of brain tumor MRIs using a kernel support vector machine." pp. 151-160.

[10] M. F. Kircher, A. de la Zerda, J. V. Jokerst, C. L. Zavaleta, P. J. Kempen, E. Mittra, K. Pitter, R. Huang, C. Campos, F. Habte, R. Sinclair, C. W. Brennan, I. K. Mellinghoff, E. C. Holland, and S. S. Gambhir, "A brain tumor molecular imaging strategy using a new triple-modality MRIphotoacoustic-Raman nanoparticle," Nature Medicine, vol. 18, no. 5, pp. 829-834, 2012/05/01, 2012.

[11] Z. N. K. Swati, Q. Zhao, M. Kabir, F. Ali, Z. Ali, S. Ahmed, and J. Lu, "Brain tumor classification for MR images using transfer learning and fine-tuning," Computerized Medical Imaging and Graphics, vol. 75, pp. 34-46, 2019/07/01/, 2019.

[12] I. Shahzadi, T. B. Tang, F. Meriadeau, and A. Quyyum, "CNN-LSTM: Cascaded Framework For Brain Tumour Classification." pp. 633-637.

[13] P. Saxena, A. Maheshwari, and S. Maheshwari, "Predictive modeling of brain tumor: A Deep learning approach," arXiv preprint arXiv:1911.02265, 2019.

[14] E.-S. A. El-Dahshan, H. M. Mohsen, K. Revett, and A.-B. M. Salem, "Computer-aided diagnosis of human brain tumor through MRI: A survey and a new algorithm," Expert Systems with Applications, vol. 41, no. 11, pp. 5526-5545, 2014/09/01/, 2014.

[15] A. Çinar, and M. Yildirim, "Detection of tumors on brain MRI images using the hybrid convolutional neural network architecture," Medical Hypotheses, vol. 139, pp. 109684, 2020/06/01/, 2020.

[16] A. Rehman, S. Naz, M. I. Razzak, F. Akram, and M. Imran, "A deep learning-based framework for automatic brain tumors classification using transfer learning," Circuits,
Systems, and Signal Processing, vol. 39, no. 2, pp. 757-775, 2020.

[17] C. Da, H. Zhang, and Y. Sang, "Brain CT Image Classification with Deep Neural Networks." pp. 653-662.

[18] X. W. Gao, R. Hui, and Z. Tian, "Classification of CT brain images based on deep learning networks," Computer methods and programs in biomedicine, vol. 138, pp. 49-56, 2017.

[19] Y. Xu, Z. Jia, Y. Ai, F. Zhang, M. Lai, I. Eric, and C. Chang, "Deep convolutional activation features for large scale brain tumor histopathology image classification and segmentation." pp. 947-951.

[20] M. Toğaçar, B. Ergen, and Z. Cömert, "BrainMRNet: Brain tumor detection using magnetic resonance images with a novel convolutional neural network model," Medical Hypotheses, vol. 134, pp. 109531, 2020.

[21] Y. Xu, Z. Jia, Y. Ai, F. Zhang, M. Lai, and E. I. Chang, "Deep convolutional activation features for large scale Brain Tumor histopathology image classification and segmentation." pp. 947-951.

[22] M. K. Abd-Ellah, A. I. Awad, A. A. M. Khalaf, and H. F. A. Hamed, "A review on brain tumor diagnosis from MRI images: Practical implications, key achievements, and lessons learned," Magnetic Resonance Imaging, vol. 61, pp. 300-318, 2019/09/01/, 2019.

[23] H. Samma, and S. A. Suandi, "Transfer Learning of Pre-Trained CNN Models for Fingerprint Liveness Detection," Biometric Systems: IntechOpen, 2020.

[24] S.-H. Wang, S. Xie, X. Chen, D. S. Guttery, C. Tang, J. Sun, and Y.-D. Zhang, "Alcoholism identification based on an AlexNet transfer learning model," Frontiers in psychiatry, vol. 10, pp. 205, 2019.

[25] T. Kaur, and T. K. Gandhi, "Deep convolutional neural networks with transfer learning for automated brain 
image classification," Machine Vision

and Applications, vol. 31, no. 3, pp. 20, 2020/03/27, 2020.

[26] Y.-D. Zhang, V. V. Govindaraj, C. Tang, W. Zhu, and J. Sun, "High performance multiple sclerosis classification by data augmentation and AlexNet transfer learning model," Journal of Medical Imaging and Health Informatics, vol. 9, no. 9, pp. 2012-2021, 2019.

[27] M. K. Abd-Ellah, A. I. Awad, A. A. M. Khalaf, and H. F. A. Hamed, "Two-phase multi-model automatic brain tumour diagnosis system from magnetic resonance images using convolutional neural networks," EURASIP Journal on Image and Video Processing, vol. 2018, no. 1, pp. 97, 2018/09/30, 2018.

[28] M. Abd-Ellah, A. I. Awad, A. A. M. Khalaf, and H. Hamed, "Deep Convolutional Neural Networks: Foundations and Applications in Medical Imaging," pp. 233-260, 2020.

[29] M. K. Abd-Ellah, A. I. Awad, H. F. A. Hamed, and A. A. M. Khalaf, "Parallel Deep CNN Structure for Glioma Detection and Classification via Brain MRI Images." pp. 304-307.

[30] M. K. Abd-Ellah, A. I. Awad, A. A. M. Khalaf, and H. F. A. Hamed, "Classification of Brain Tumor MRIs Using a Kernel Support Vector Machine." pp. 151-160.

[31] Z. N. K. Swati, Q. Zhao, M. Kabir, F. Ali, Z. Ali, S. Ahmed, and J. Lu, "Content-Based Brain Tumor Retrieval for MR Images Using Transfer Learning," IEEE Access, vol. 7, pp. 17809-17822, 2019.

[32] ImageNet, "http://www.imagenet.org."

[33] K. Clark, B. Vendt, K. Smith, J. Freymann, J. Kirby, P. Koppel, S. Moore, S. Phillips, D. Maffitt, M. Pringle, L. Tarbox, and F. Prior, "The Cancer Imaging Archive (TCIA): Maintaining and Operating a Public Information Repository," Journal of Digital Imaging, vol. 26, no. 6, pp. 
Vol. 41, No.2. July 2022

الكثف التلقائي عن أورم المخ من خلال صور التصوير بالرنين المغناطيسي باستخدام نموذج نقل التعلم مع الثبكة العصبية التلافيفية العميقة

إسر اء صلاح بيومي 1، محمود خالد عبداللاه 2*، اشرف عبدالمنعم خلف 3، رضا رجب غريب 4

1 باحث ، معيدة بقسم هندسة الاتصالات و الاكترونيات ، الاكاديمية المصرية للهندسة والتكنولوجيا المتقدمه ، القاهرة ، مصر.

2مدرس بقسم هندسة الاتصالات و الالكترونيات ، معهد المدينة العالى للهندسة و التكنولوجيا ، الجيزة ، مصر.

3استاذ و رئيس قسم الهندسة الكهربية ، كلية الهندسة ، جامعة المنيا ، المنيا ، مصر.

4استاذ بقسم الهندسة الكهربية ، كلية الهندسة ، جامعة أسيوط ، أسيوط ، مصر.

: (الملخص :

إن الاكتشاف المبكر للورم الدماغي له دور هام في تحسين علاج المريض و ابقائه على قيد الحياة. يعد تقييم صور التصوير بالرنين المغناطيسي (MRI) يدويا مهمة صعبة للغاية بسبب أعداد الصور الكثيرة التي يتم تصويرها للمرضى في المستشفيات بشكل روتيني. ولذللك، فإن هناك حاجة لاستخدام نظام التشخيص بمساعدة الكمبيوتر (CAD) للكثف المبكر عن أورام المخ وتصنيفها على أنها طبيعية وغير طبيعية. يهدف البحث إلى تصميم وتقييم نظام المطبق على الشبكة العصبية الالتفافية (CNN) الحديثة والمقترحة على مدار السنوات الأخيرة. تم تطبيق خمسة تعديلات مختلفة على خمسة شبكات CNN مشهورة و عالمية لمعرفة التعديل الأكثر فعالية. ينم تطبيق التعديلات على خمسة طبقات مختلفة مع ضبط معايير كل بنية لتقديم شبكة عصبية التفافية جديدة للكثف عن ورم الدماغ. تحتوي معظم بيانات أورام المخ على عدد صغير من الصور لتدريب بنية التعلم العميق. لذلك، يتم استخدام مجمو عتي بيانات في التقييم لضمان فعالية التصميم المقترحة. أولاً، مجموعة مكونه من 120 صورة بالرنين المغناطيسي للمخ تتضمن 60 صورة غير طبيعية و 60 صورة طبيعية. ثانيًا، مجموعة صور قياسية من قاعدة بيانات RIDER Neuro MRI تتضمن 349 صورة تصوير بالرنين المغناطبسي للمخ، تحتوى على 109 صورة طبيعية و 240 صورة غير طبيعية. نظهرالنتائج أن نظام CNN Transfer Learning المقترح باستخدام التصوير بالرنين المغناطيسي يمكنه التعرف على المؤشرات الحيوية المهمة لورم الدماغ. كما يظهر أن أفضل دقة ونوعية وحساسية تم الحصول عليها هي 100٪ لكل منهم. 